\title{
El tamoxifeno es beneficioso en la mayoría de las pacientes con cáncer mamario temprano
}

Tamoxifen for early breast cancer: an overview of the randomised trials. Early Breast Cancer Trialists Collaborative Group. Lancet 1998; 351: 1451-1466.

\section{Objetivo}

Revisar la evidencia científica en relación al beneficio del tamoxifeno en cuanto a reducción de recurrencias, de la mortalidad, y evaluar seguridad y duración del tratamiento en pacientes con cáncer de mama temprano.

\section{Fuente y selección de datos}

El grupo colaborativo contra el cáncer de mama procesó todos los datos publicados al respecto en estudios a leatorizados desde 1984 a 1996. Los trabajos analizados fueron divididos según la duración del tratamiento con tamoxifeno en tres categorías: 1 ; 2 ; y 5 años. Se identificaron 63 estudios, 55 de ellos cumplieron criterios de inclusión (involucrando 37.000 mujeres).

La información recolectada incluyó: edad, menopausia, compromiso ganglionar, medición de receptores hormonales, fechas de a leatorización, de tratamiento, aparición de cáncer contralateral o segundas neoplasias, recurrencia local o a distancia, muerte y sus causas.

\section{Resultados principales}

De las 37.000 mujeres, en las $\sim 8.000$ que tenían receptores estrogénicos negativos (RE-), el beneficio del tamoxifeno fue pequeño (RRR* $10 \%$, IC95\% 2-17\%, $\mathrm{p}<0.001$ ). En el resto de las mujeres (18.000 con $\mathrm{RE}+\mathrm{y} 12.000$ con RE no evaluados) los beneficios fueron estadística y clínicamente relevantes. Los siguientes datos se refieren a este grupo de 30.000 pacientes. En 10 años de seguimiento la reducción en las recurrencias fueron $21 \%, 29 \%$ y $47 \%$ en los grupos de tratamiento con tamoxifeno de 1, 2, y 5 años respectivamente y la reducción en la mortalidad global fue de $12 \%, 17 \%$ y $26 \%$ respectivamente. Tanto la recurrencia como la mortalidad disminuían a medida que se incrementaban los años de tratamiento ( $P<0.003$ para ambas).

La reducción relativa en la mortalidad fue similar tanto en mujeres con o sin compromiso ganglionar, pero las reducción absoluta de la mortalidad fue mayor en mujeres con compromiso axilar positivo. La reducción absoluta de recurrencias osciló entre un $4.7 \%\left(N N T^{*}=21\right)$ en estudios de un año y mujeres con ganglios negativos y un $15.2 \%$ (NNT=7) en estudios de cinco años y mujeres con ganglios positivos. La reducción absoluta de la mortalidad osciló entre un 3.4\% (NNT=30) en estudios de un año y mujeres sin ganglios y un $10.9 \%$ (NNT=9) en estudios de cinco años y mujeres con ganglios positivos. En todas las mujeres analizadas (incluyendo a las ER -) la reducción relativa en la aparición de cáncer de mama contralateral fue de $13 \%$, 26\% y $47 \%$ en los trabajos con 1, 2, 5 años de tamoxifeno respectivamente. La densidad de los receptores de estrógenos se relacionó directamente con mejoría en la sobrevida y las recurrencias. La posibilidad de una respuesta predecible al tamoxifeno no se relacionó con la presencia de receptores de progesterona, edad, menopausia, dosis, o si recibieron quimioterapia.

La incidencia de cáncer de endometrio fue aproximadamente el doble en los grupos con 102 años de tamoxifeno y el cuádruple en el grupo con 5 años de tratamiento. El exceso de riesgo absoluto promedio fue de 4 casos por 1000 pacientes a diez años $\left(N N D^{*}=250\right.$, es decir que se provocaría un cáncer de endometrio cada 250 mujeres tratadas a los diez años). La reducción absoluta en la incidencia de cáncer de mama contralateral fue casi el doble que el incremento absoluto en la incidencia de cáncer de endometrio. El tamoxifeno no aumentó las muertes por cáncer de colon u otras causas.

\section{Conclusiones}

El tamoxifeno como tratamiento adyuvante del cáncer de mama temprano mejora la sobrevida y disminuye las recurrencias en pacientes con receptores estrogénicos positivos o desconocidos. En pacientes con receptores negativos todavía es necesario seguir las investigaciones con estudios controlados.

\section{COMENTARIO}

Ya se ha demostrado que el uso de tamoxifeno adyuvante mejora la sobrevida a 10 años, sin embargo aún quedaban preguntas por responder: ¿Quiénes deben ser tratados?, ¿Por cuánto tiempo?, ¿Los riesgos del uso superan los beneficios en mujeres con bajo riesgo de recurrencia? El tamoxifeno reduce las recurrencias y la mortalidad en un $20-40 \%$ dependiendo de las características del tumor ${ }^{1}$. Las pacientes con tumores verdaderamente ER- no parecen beneficiarse con el tamoxifeno, pero si aquellas con ER+ (especialmente aquellos con alta intensidad).

Tanto las mujeres pre y postmenopausicas, como aquellas con axila positiva o no, se benefician con el tamoxifeno si poseen ER+. Esto nos hace pensar en cambiar algunos algoritmos de tratamientos que ya parecían estar fuertemente establecidos. La duración óptima del tratamiento con tamoxifeno parece ser de 5 años. Faltan estudios con adyuvancia hormonal más prolongada.

Una complicación poco frecuente es el cáncer de endometrio de bajo grado (en particular en post-menopáusicas), pero el riesgo por su aparición no llega a contrarrestar los beneficios en la sobrevida y de prevención de recurrencias.

Este importante, exhaustivo y meticuloso meta-análisis no demostró aumento de la incidencia de otras neoplasias o mortalidad por otras causas.

Una pregunta importante es si las mujeres pre-menopáusicas con ER+ deben ser tratadas con quimioterapia, tamoxifeno o ambos. Sólo un pequeño estudio demostró que el esquema Ciclofosfamida-Metrotexate-Fluouracilo fue superior al tamoxifeno. Otros trabajos no hallaron beneficios usando tamoxifeno luego de quimioterapia en pre-menopáusicas.

En post-menopáusicas, el tamoxifeno es superior a la quimioterapia, pero el interrogante es si la quimioterapia puede adicionar un beneficio adicional. Este meta-análisis sugiere que el agregado de quimioterapia provee un pequeño incremento de la reducción en recurrencias y mortalidad comparado con el tamoxifeno solamente. Resta evaluar si este beneficio marginal supera los efectos adversos, costos, y cómo esto afecta la calidad de vida de las mujeres con esta enfermedad. 